\title{
Childhood rhabdomyosarcoma metastatic to bone marrow presenting with disseminated intravascular coagulation and acute tumour lysis syndrome: review of the literature apropos of two cases
}

\author{
Ewa Bien • Lucyna Maciejka-Kapuscinska $\cdot$ Maciej Niedzwiecki • \\ Joanna Stefanowicz • Anna Szolkiewicz • Malgorzata Krawczyk • \\ Jadwiga Maldyk • Ewa Izycka-Swieszewska • Beata Tokarska • \\ Anna Balcerska
}

Received: 1 March 2010/Accepted: 10 May 2010/Published online: 2 June 2010

(C) The Author(s) 2010. This article is published with open access at Springerlink.com

\begin{abstract}
The paper presents diagnostic and therapeutic difficulties in two adolescents with widespread rhabdomyosarcoma (RMS) presenting with severe haemorrhages resulting from disseminated intravascular coagulation (DIC) and with laboratory features of acute tumour lysis syndrome (ATLS). Other published cases of childhood RMS with DIC at admission have been listed and reviewed. It has been concluded that the clinical picture of a widespread RMS in children may resemble acute hematologic malignancy and pose a big diagnostic problem. That is why the presence of small blue round cells morphologically similar to lymphoblasts and/or myeloblasts in bone marrow (BM), lacking hematopoietic makers, should prompt the pathologist to consider possible diagnosis of RMS. Inclusion of desmin, MyoD1 and myogenin Myf4 to the immunohistochemical panel is obligatory in such cases. When the
\end{abstract}

E. Bien $(\square) \cdot$ L. Maciejka-Kapuscinska · M. Niedzwiecki J. Stefanowicz - A. Szolkiewicz · M. Krawczyk · A. Balcerska Department of Paediatrics, Haematology, Oncology and Endocrinology, Medical University of Gdansk, 7 Debinki Street, 80-211 Gdansk, Poland

e-mail: ewabien1@wp.pl

J. Maldyk

Department of Childhood Pathomorphology,

Medical University of Warsaw, Warsaw, Poland

E. Izycka-Swieszewska

Department of Pathomorphology, Medical University of Gdansk, Gdansk, Poland

B. Tokarska

Department of Radiology, Medical University of Gdansk, Gdansk, Poland representative histopathological tumour specimens are difficult to obtain, the flow cytometric immunophenotyping of BM metastases could help the standard morphological/ immunohistological diagnostic procedures and advance the diagnosis. Recently, the flow cytometric CD45- CD56+ immunophenotype together with Myf4 transcript has been assigned to RMS cells infiltrating BM. In children with disseminated RMS complicated with DIC rapid polychemotherapy aimed at diminishing the malignancy-triggered procoagulant activity should be initiated. However, in cases with concomitant ATLS the initial doses of chemotherapy should be reduced and the metabolic disorders and renal function monitored. The prognosis in children with RMS metastatic to BM with signs of DIC or ATLS at admission depends on the response to chemotherapy, however generally it is highly disappointing.

Keywords Acute tumour lysis syndrome .

Bone marrow metastasis - CD45- CD56+ immunoprofile . Childhood rhabdomyosarcoma $\cdot$ Disseminated intravascular coagulation · Flow cytometry $\cdot$ Review

\section{Introduction}

Disseminated intravascular coagulation (DIC), also known as consumption coagulopathy, is an abnormal activation of coagulation cascade that occurs in response to different diseases. It accounts for up to $1 \%$ of hospitalized patients and may happen in a wide variety of severe medical conditions, including sepsis, trauma, obstetrical complications, transfusion and toxic reactions, rheumatologic illnesses, vascular abnormalities and also in neoplasms [1]. 
The association between neoplasms and DIC is wellknown and has been described in both solid and hematologic malignancies. DIC has been reported to be present in approximately $15-20 \%$ of patients with acute leukaemia (up to $90 \%$ in acute promyelocytic leukaemia, APL) [2] and in $7-15 \%$ of patients with metastatic solid tumours [3-6]. The mechanisms leading to DIC in patients with cancer are unclear [1,7]. Experimental studies indicate that the solid tumour and injured endothelial cells may release mediators with a procoagulant activity (i.e., tissue factor and cancer procoagulant) [8] or, alternatively, induce a hypofibrinolytic state mostly due to high levels of the fibrinolytic inhibitor PAI-1 [9]. Virtually all pathways that contribute to the occurrence of DIC are driven by proinflammatory cytokines such as tumour necrosis factor, interleukin-1 $\beta$, interleukin-6 and interleukin-8 $[10,11]$.

Patients with malignancy-driven DIC usually demonstrate thrombocytopenia, hypofibrinogenemia, and elevated serum levels of D-dimers and fibrinogen degradation products $[3,12]$. They may, however, be asymptomatic despite demonstrable laboratory features of excess thrombin generation, which has been referred to as chronic or low-grade DIC [7, 13]. Chronic systemic activation of coagulation can, eventually, lead to exhaustion of platelets and coagulation factors. Thus bleeding may be the first clinical symptom indicating the presence of DIC. Severe bleeding episodes may also result from the activation of the fibrinolytic system which predominates in metastatic prostate cancer, pancreatic adenocarcinoma or APL $[3,4,14,15]$.

Very uncommonly, patients with rhabdomyosarcoma (RMS) may present with clinical and/or laboratory features of DIC. Such presentations were seen mainly in widespread alveolar RMS metastatic to BM [16]. Only few reports on RMS with DIC are available in the literature. Here we describe two cases of widespread RMS in adolescents with clinical presentation of severe haemorrhages resulting from DIC. Both patients demonstrated also the laboratory features of acute tumour lysis syndrome (ATLS) which, together with DIC, were strongly suggestive for acute hematologic malignancy and exceptionally rare in RMS course [17].

\section{Patients' characteristics}

\section{Patient 1}

A 14-year-old boy was admitted to the Department of Paediatric Oncology, Medical University of Gdansk, Poland in February 2007 suspected of acute leukaemia. For 2 weeks he had demonstrated severe back and abdominal pains, fever, pallor, increasing skin diathesis and enlarged lymph nodes of the neck. At admission the patient's general state was poor with signs of: anaemia, weakness, cachexia and disseminated abundant haemorrhagic petechiae and ecchymoses. Left retroauricular and bilateral inguinal lymphadenopathy and slight hepato-splenomegaly were found. During the 1st days of hospitalization he developed new enlarged occipital lymph nodes and multiple small $(2-5 \mathrm{~mm})$ nodules in the subcutaneous tissue of chest, abdomen and back. He demonstrated dyspnoea due to increasing volume of the bilateral pleural fluid. Computed Tomography (CT) additionally revealed huge mediastinal lymphatic masses and significantly enlarged lymph nodes in the spleen hilus and left kidney as well as ascites and multiple metastatic nodules within peritoneum (Fig. 1). Laboratory tests showed anaemia, thrombocytopenia and leucocytosis with left shift, ESR and C-reactive protein values were slightly elevated. Laboratory signs of ATLS (LDH > 1,068 U/l, uric acid $9.3 \mathrm{mg} / \mathrm{dl}$, phosphorus $5.2 \mathrm{mg} / \mathrm{dl}$ ) and DIC (listed in Table 1) were present.

The repeated myelograms revealed massive dyserythropoietic features. Atypical cells constituted more than $50 \%$ of the BM cellularity and suggested strongly leukemic blasts by morphology (Fig. 2). In flow cytometry (FC), however, the population of atypical cells did not display antigens characteristic for the lymphoid and myeloid cell lines. They were found CD45 negative (Fig. 3) and also CD61, CD62, CD71 and CD235a negative. Normal populations of BM cells were found mostly within the CD45+ gate, apart from the erythroblasts, which were CD45 negative, but CD71 and CD235a positive in FC. Similar immunophenotype of the metastatic cells was detected later on in FC of the haemorrhagic pleural effusion. The atypical cells displayed abnormal karyotype with partial trisomy of chromosome 2 and partial tetrasomy of chromosome 6 $(48, \mathrm{XY},+\operatorname{dic}(2 ; 6)(\mathrm{q} 21 ; \mathrm{p} 21),+6[6])$. FISH examination of $\mathrm{BM}$ did not detect the PML/RARA gene fusion.

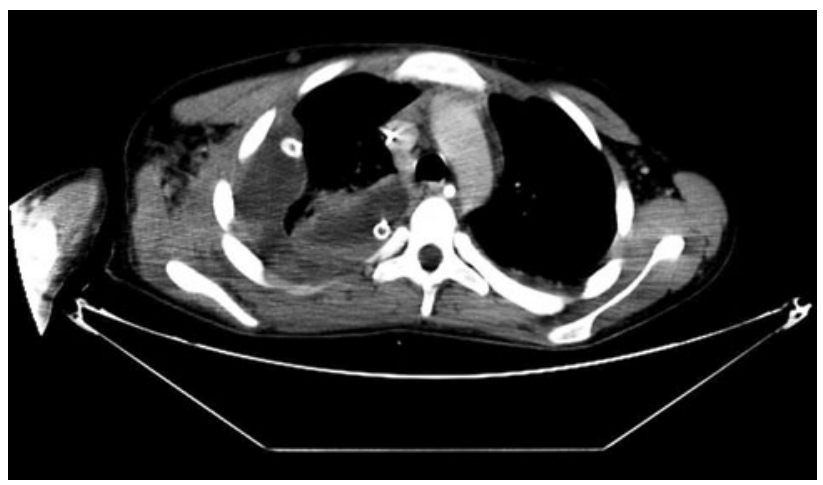

Fig. 1 CT scan of the chest of the patient 1 revealing large amount of free liquid in right pleural cavity with drainage and a pathological nodule in subcutaneous tissue within the right anterior part of chest wall 


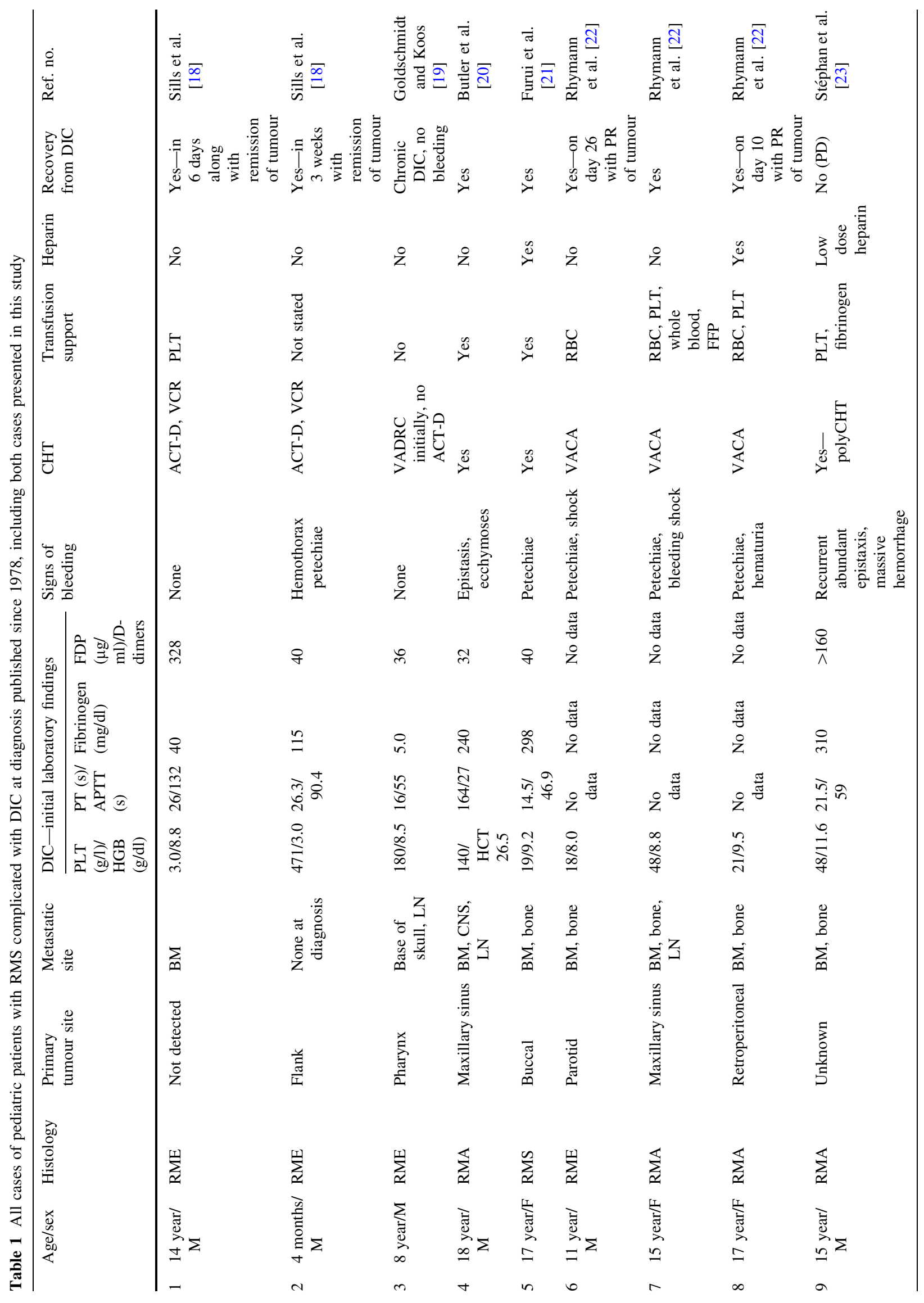




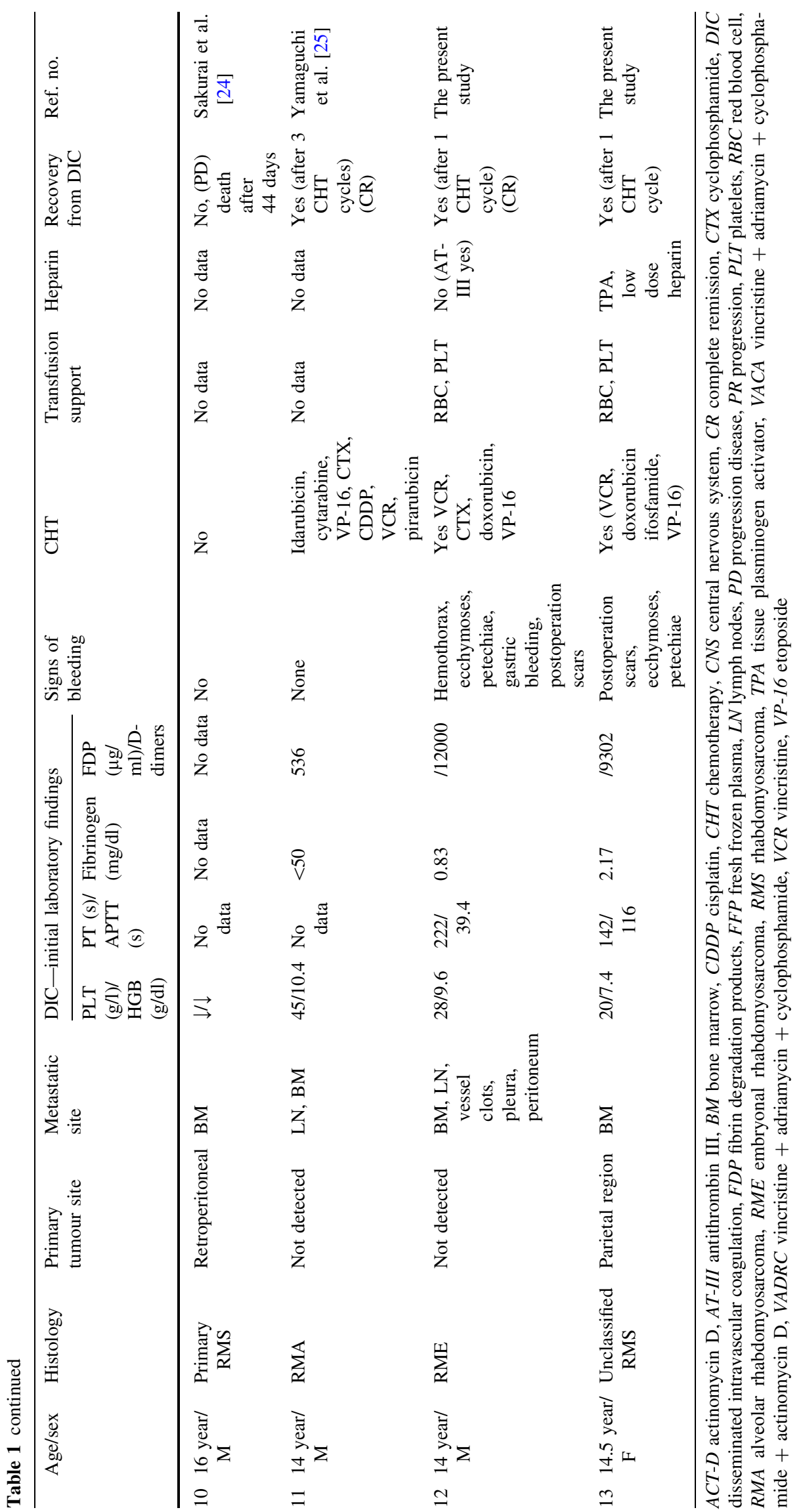




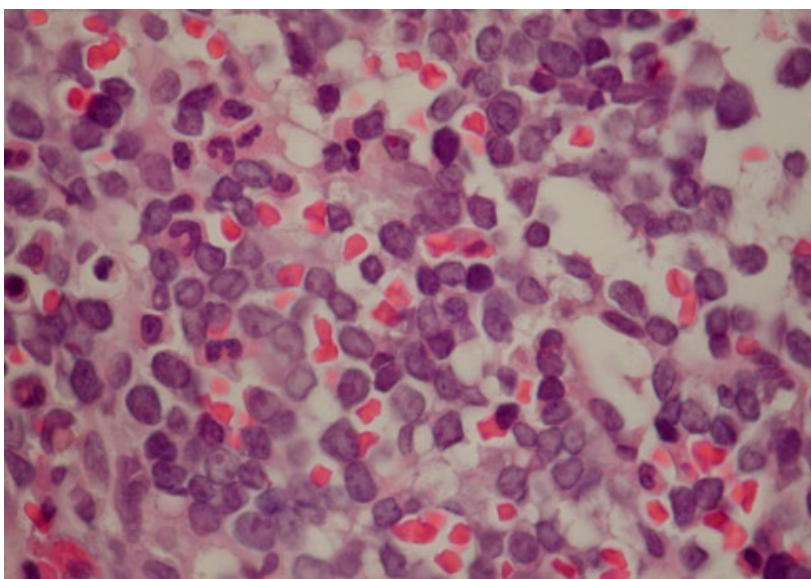

Fig. 2 BM smear of the patient 1 with extensive infiltration by a homogeneous population of the primitive malignant small round blue cells (haematoxylin and eosin stain, low-power view). These cell were desmin positive and represented BM RMS metastases. Residual hematopoietic cells are visible at the left upper quadrant of the picture

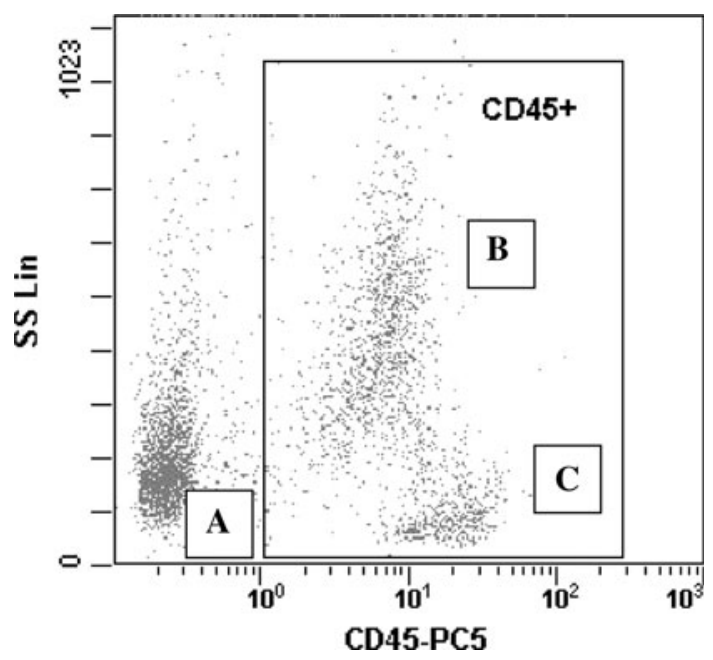

Fig. $3 \mathrm{FC}$ of the $\mathrm{BM}$ of patient 1 revealed the presence of a population of CD45 negative cells $(A)$, constituting more than $50 \%$ of the $\mathrm{BM}$ cells (this corresponded to the result of the morphologic examination of BM). These atypical cells were diagnosed as BM metastases of a malignant nonhematopoietic tumour because they did not display antigens characteristic for lymphoid or myeloid cell lines, being CD61, 62, 71 and 235a negative. Normal BM cells were found mostly within the CD45+ gate (neutrophils- $B$, lymphocytes- $C$ ), apart from the erythroblasts, which were CD45 negative, but also CD71 and CD235a positive in FC

The surgical biopsy of the retroauricular lymph node and two subcutaneous nodules was performed. Histopathologically the sections demonstrated in part necrotic neoplastic infiltrates made of small round and oval tumour cells similar to those seen in the BM aspirate. The cells were arranged in small lobules. Based on H-E staining the initial diagnosis of lymphoma was made, which was changed after the narrow introductory immunohistochemical panel results into Ewing sarcoma. Because of the fast neoplasm's progression and increasing massive right hemothorax resulting from uncontrollable DIC, the patient was given life-saving chemotherapy (CHT) despite the lack of definite histopathologic and cytologic diagnosis. The patient received treatment effective in both malignant non-Hodgkin's lymphoma and Ewing sarcoma, including: corticosteroids, vincristin, etoposide, adriamycin and cyclophosphamide. Up to 2,000 $\mathrm{ml}$ of haemorrhagic fluid a day was drained from the right pleural cavity (Fig. 1). The patient was also bleeding from postoperational scars, injection spots and stomach. D-dimers increased up to 12,000 and $\mathrm{Hb}$ and PLT decreased to $7.3 \mathrm{~g} / \mathrm{dl}$ and $15 \mathrm{~g} / \mathrm{l}$, respectively. The patient required repeated blood products transfusions and substitution of AT-III. Despite therapy, massive hemothorax caused mediastinal displacement and urgent right thoracotomy was performed disclosing huge haematoma of the lower lobe of the right lung and neoplastic infiltration of the whole pleura. The biopsy from pleural infiltration was taken and sent to referral histopathologic centre. The patient was transferred to the Emergency Unit and treated with mechanical ventilation aimed to expand the right lung and possibly diminished pleural bleeding.

After 3 days unified diagnosis of disseminated RMS of most probably embryonal type with unknown primary site was made. The patient was in extremely poor condition, however we decided to introduce reduced doses of vincristine, cyclophosphamide and actinomycin-D. After 2 days DIC parameters started to improve and bleeding and skin diathesis gradually diminished. The lymphatic masses started to shrink and the patient' general state improved. After 6 days the patient was extubated and then continued CHT according to CWS-2002 protocol for childhood soft tissue sarcomas. CT and myelogram examinations revealed complete remission of the disease after two cycles of CHT. Unfortunately, after the sixth one the patient demonstrated severe neurologic abnormalities (chorea, ataxia, headaches). MRI of the brain showed multiple cortical and subcortical metastatic foci with surrounding vasogenic oedema. Despite aggressive therapy the patient died of disease progression 3 months later.

\section{Patient 2}

A 14.5-year-old girl was admitted to our department in January 2009 suspected of hematologic malignancy. For 2 weeks she had demonstrated poor general condition, weakness, and severe headaches and pains of bones and of the right inguinal area. She lost weight $(10 \mathrm{~kg})$ during the previous 4 months. Abundant petechiae end ecchymoses were present on the upper and lower limbs. Huge $(9 \times 3.5 \times 9 \mathrm{~cm})$ tumour of the left parietal region and slight hepato-splenomegaly were found in physical 
Fig. 4 Bone marrow of the patient 2 with big atypical tumour cells with marked cytoplasmic vacuolation (May-Grunwald/Giemsa stain, high power view), sharing some similarities with lymphoblasts. The cells were scattered, sometimes gathered in small clusters

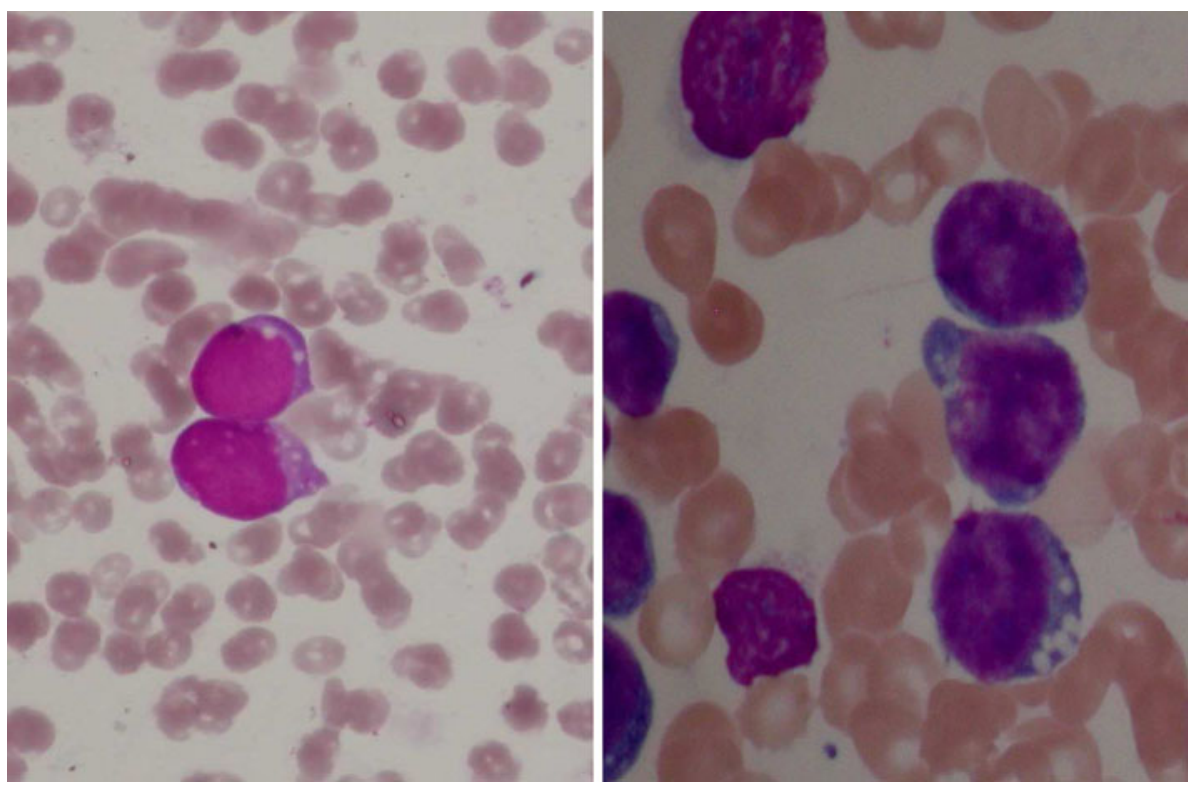

examination. In CT the tumour involved soft tissues and the parietal bone. Laboratory tests revealed markedly decreased platelet and haemoglobin levels and features suggestive for DIC (Table 1) and ATLS (LDH 2,971 U/1, uric acid $10.4 \mathrm{mg} / \mathrm{dl}$, phosphorus $5.3 \mathrm{mg} / \mathrm{dl}$ ). In myelogram the population of atypical cells accounting for over $50 \%$ of BM was stated. By morphology they resembled leukemic blasts (Fig. 4); in FC they were CD45 negative. The cytogenetic analysis of atypical cells present in peripheral blood displayed abnormal karyotype with multiple structural and numerical abnormalities (90-94,XXXX,der(2)t $(2 ; 11)$ $(\mathrm{q} 35 ; \mathrm{q} 13),+\operatorname{del}(5)(\mathrm{q} 11) \times 2, \operatorname{add}(6)(\mathrm{q} 13)[2],-10,+\operatorname{der}(12) \mathrm{t}$ $(1 ; 12)(\mathrm{q} 23 ; \mathrm{q} 13),-13,-13,-14,-16,+17, \operatorname{mar} 1, \operatorname{mar} 2, \operatorname{mar} 3$,inc [cp20]).

The tissue material from the first neurosurgical biopsy was not representative and devoid of neoplastic tissue, so the repeated sampling was necessary. Before that the condition of the patient dramatically worsened. She demonstrated symptoms of increased intracranial pressure confirmed in eye fundus examination. Performed CT images were irrelevant. The CNS symptoms resolved after anti oedematous therapy and fluids restriction. On the next day severe dyspnoea with lowered alveolar sound over the right lung occurred and the disseminated pulmonary thromboembolism was stated. The patient received tissue plasminogen activator in 48-h-infusion and subsequently, low-molecular-weight heparins subcutaneously, with a good clinical effect. Anticoagulative therapy resulted in massive haemorrhage from the postoperational scar in the parietal area and exacerbation of the skin diathesis, requiring multiple blood transfusions. That is why the second diagnostic biopsy of the parietal tumour had to be postponed and instead, life-saving CHT was introduced.
Because no histopathologic diagnosis was available at that moment and clinical symptoms suggested mostly Ewing sarcoma, the patient received cycle of EURO-EWING 99 protocol, including vincristin, doxorubicin, ifosfamide and etoposide. The doses were reduced because of poor general state of the patient and ATLS features.

Finally, the histopathological diagnosis was established upon the examination of trephine biopsy and then confirmed in the material obtained from the second neurosurgical parietal tumour biopsy performed 3 weeks after the first surgical attempt. The tissue material was scanty and in part necrotic. The oval and spindled neoplastic cells were dispersed in post chemotherapy regressively changed tissue. The immunophenotype: desmin+, MyoD1+, Myf 4+, $\mathrm{S}-100+$, corresponded to the diagnosis of RMS of unclassified subtype. The child was given CWS-2006 protocol for metastatic paediatric RMS and DIC signs disappeared after the second cycle of CHT. Complete remission has been stated since the third cycle until March 2010, when the relapse in the occipital region has been stated.

\section{Discussion}

DIC is a rare feature of paediatric solid malignancies even in metastatic phase. It has been reported in disseminated neuroblastoma [26], nephroblastoma [27], infantile fibrosarcoma [28] and multi-system Langerhans cell histiocytosis [29]. However, most cases have been associated with advanced RMS presenting with BM metastases [30]. Table 1 summarizes all cases of paediatric RMS presenting with DIC published from 1978 till now, including both 
cases presented herein. All these patients were reported to fulfil the clinical and/or laboratory criteria of DIC. Most of them were adolescents and males. In contrast to adult series published previously, we have not stated a predominance of alveolar subtype of RMS (RMA) in this group [30, 31]. This may result from the fact that RMA is less common type of RMS in children and the most popular in adults. Some authors have speculated that RMA disseminated to $\mathrm{BM}$ is typically associated with hypercoagulability and/or hyperfibrinolysis due to the presence of pathognomonic translocation, $\mathrm{t}(2 ; 13)(\mathrm{q} 35 ; \mathrm{q} 14)[30,31]$.

The clinical utility of cytogenetic studies in the differential diagnostics of malignant small cell round tumours in children has been reported and emphasized [32-34]. Accordingly, Stéphan et al. [32] have been able to diagnose disseminated RMA in a 15-year-old boy based on the presence of this typical translocation [32]. However, other than RMA types of childhood RMS lack any typical cytogenetic abnormality. Our patients were diagnosed with multiple but not prognostic chromosomal abnormalities detected within the neoplastic cells. Therefore the proper diagnoses of our patients (particularly of the patient 1) were markedly delayed. The clinical symptoms of both patients were suggestive for acute hematologic malignancies, especially that the clinical and laboratory features of DIC and ATLS were present. The presence of ATLS in RMS is exceptionally rare [17] and there have been no cases with both DIC and ATLS among childhood RMS published to date.

By the nature of their morphology and biology, small round cell tumours, particularly RMA may infiltrate the marrow and can be misdiagnosed as acute leukemias. Also clinical picture of disseminated RMS may sometimes resemble acute hematologic malignancy, especially when clinical and/or laboratory signs of DIC and ATLS occur and no discernible primary lesion is found [34-36]. In our patients serious problems to obtain representative diagnostic material occurred and both had to be biopsied once more which was extremely risky because of DIC-associated-bleeding from incisional scars. There were several conflicting initial diagnoses, including haematological and non-haematological malignancies before, eventually; the final diagnoses of RMS were established after the consultations of the specimens in three different histopathological centres. It appears that FC immunophenotyping of BM atypical cells is a valuable and available diagnostic tool in such cases. By this method we were able to exclude the diagnosis of leukemias and lymphoma by stating that the metastatic cells were CD45 negative and did not display any surface antigens characteristic for lymphoid or myeloid cell lines. CD45 expression is regularly lower on leukemic blasts than on normal lymphoid and myeloid cells, but
CD45 negative leukemias are extremely rare and the atypical cells were CD235a negative, which excluded erythroleukemia.

Unfortunately, we were not able to perform the CD56 phenotyping. Recently, the FC CD45- CD56+ immunoprofile together with Myf4 transcript have been assigned to RMS cells infiltrating BM [37]. The immunoprofile CD45- CD56+ alone is not enough to identify the RMS cells as it is present in many types of nonhematopoietic small round blue cell tumours, including neuroblastoma, neuroendocrine and mesenchymal tumours [38].

The prognosis in RMS metastatic to BM is very poor especially in cases complicated with DIC. Thus it is of utmost importance to introduce early polychemotherapy aimed at diminishing the malignancy-triggered procoagulant activity [39] even if the definite histopathologic diagnosis is inconclusive. In an inoperable situation, an immediate beneficial effect has been stated upon administration of multidrug CHT, even if a measurable antitumor effect is not observed [40]. In most patients summarized in Table 1 such life-saving, individual treatment contained cytostatics acting in different phases of the malignant cell proliferation cycle [41]. That is why we introduced CHT immediately, despite inconclusive diagnosis and poor patients' condition with massive acute bleedings. Fortunately both patients responded well to the treatment and DIC symptoms significantly diminished after the first and disappeared after the second cycle of CHT.

Once the treatment has been directed at the primary illness, the additional therapy of DIC itself may be added. In patients listed in Table 1 neither treatment of cancer nor the supportive management of DIC was homogeneous. Generally, the routine platelet replacement is not indicated in acute DIC unless ongoing bleeding is present or invasive procedures are planned [42]. In patient 1 massive haemorrhage from the pleura required as many as 57 units of erythrocytes and 69 units of platelets transfusions. Patient 2 was transfused during haemorrhage from postoperational scar, however she developed anti-platelet antibodies and was not supplemented with platelets despite very low levels. The role of high- or low-dose heparin therapy in malignancy-associated DIC is still controversial, however it is indicated in cases with obvious thromboembolic disease or where fibrin deposition predominates [43, 44]. The majority of patients listed in Table 1 did not receive heparin treatment. In our material patient 2 needed immediate anticoagulating for pulmonary thromboembolism, however it resulted in massive hemorrhagic complications. In both patients clinical and laboratory signs of DIC vanished along with good response to CHT. Similarly, among the patients presented in Table 1 good response to CHT was associated with fast disappearance of the DIC symptoms. 


\section{Conclusion}

The clinical picture of a wide-spread RMS may resemble acute hematologic malignancy and pose a big diagnostic problem, especially when patients present with clinical and/or laboratory signs of DIC and ATLS. The presence of cells morphologically similar to lymphoblasts and/or myeloblasts in the BM, lacking hematopoietic makers, should prompt the hematopathologist to consider possible diagnosis of RMS. Inclusion of desmin, MyoD1 and Myf4 to the immunohistochemical panel is obligatory in such cases. When the representative histopathological tumour specimens are difficult to obtain, the FC immunophenotyping of BM metastases could help the standard morphological/ immunohistological diagnostic procedures and advance the diagnosis. Recently, the flow cytometric CD45CD56+ immunophenotype together with Myf4 transcript has been assigned to RMS cells infiltrating the BM. Because of the extremely poor prognosis in children with disseminated RMS complicated with DIC and ATLS, proper polychemotherapy aimed at diminishing the malignancy-triggered procoagulant activity should be initiated as soon as possible.

Open Access This article is distributed under the terms of the Creative Commons Attribution Noncommercial License which permits any noncommercial use, distribution, and reproduction in any medium, provided the original author(s) and source are credited.

\section{References}

1. Levi M, de Jonge E, van der Poll T (2004) New treatment strategies for disseminated intravascular coagulation based on current understanding of the pathophysiology. Ann Med 36: 41-49

2. Avvisati G, Lo Coco F, Mandelli F (2001) Acute promyelocytic leukemia: clinical and morphologic features and prognostic factors. Semin Hematol 38:4-12

3. Sallah S, Wan JY, Nguyen NP et al (2001) Disseminated intravascular coagulation in solid tumors: clinical and pathologic study. Thromb Haemost 86:828-833

4. Duran I, Tannock IF (2006) Disseminated intravascular coagulation as the presenting sign of metastatic prostate cancer. J Gen Intern Med 21:C6-C8

5. Oh WK (2002) Hematologic complications of prostate cancer. Prostate cancer, principles and practice. Lippincott Williams and Wilkins, Philadelphia

6. De la Fouchardiere C, Flechon A, Droz JP (2003) Coagulopathy in prostate cancer. Neth J Med 61:347-354

7. Okajima K, Sakamoto Y, Uchiba M (2000) Heterogeneity in the incidence and clinical manifestations of disseminated intravascular coagulation: a study of 204 cases. Am J Hematol 65: 215-222

8. Levi M, de Jonge E, van der Poll T et al (2001) Advances in the under standing of the pathogenetic pathways of disseminated intravascular coagulation result in more insight in the clinical picture and better management strategies. Semin Thromb Hemost 27:569-575
9. Ishibashi M, Ito N, Fujita M et al (1994) Endothelin-1 as an aggravating factor of disseminated intravascular coagulation associated with malignant neoplasms. Cancer 73:191-195

10. Levi M (2001) Cancer and DIC. Haemostasis 31:47-48

11. Stouthard J, Levi M, Hack CE et al (1996) Interleukin-6 stimulates coagulation but not fibrinolysis in humans. Thromb Haemost 76:738-742

12. Taylor FB Jr, Toh CH, Hoots WK et al (2001) Towards definition, clinical and laboratory criteria, and a scoring system for disseminated intravascular coagulation. Thromb Haemost 86: $1327-1330$

13. Levi M (2004) Systemic microangiopathies in the cancer patient. In: Lugassy G (ed) Thrombosis and cancer. Taylor and Francis, London

14. Navarro M, Ruiz I, Martín G et al (2006) Patient with disseminated intravascular coagulation as the first manifestation of adenocarcinoma of the prostate. Risks of prostatic biopsy. Prostate Cancer Prostatic Dis 9:190-191

15. Uchiumi H, Matsushima T, Yamane A et al (2007) Prevalence and clinical characteristics of acute myeloid leukemia associated with disseminated intravascular coagulation. Int J Hematol 86: 137-142

16. Bick RL (1992) Coagulation abnormalities in malignancy: a review. Semin Thromb Hemost 18:353-372

17. Khan J, Broadbent V (1993) Tumor lysis syndrome complicating treatment of widespread metastatic abdominal rhabdomyosarcoma. Pediatr Hematol Oncol 10:151-155

18. Sills RH, Stockman JA III, Miller ML et al (1978) Consumptive coagulopathy. A complication of therapy of solid tumors in childhood. Am J Dis Child 132:870-872

19. Goldschmidt B, Koos R (1980) Low intensity intravascular coagulation in rhabdomyosarcoma. Orv Hetil 121:905-907

20. Butler WM, Sciala SJ, Taylor HG (1982) Alveolar rhabdomyosarcoma associated with disseminated intravascular coagulation and a unique factor VIII antigen. Arch Intern Med 142:1379-1380

21. Furui T, Ichihara K, Ikeda A et al (1983) Subdural hematoma associated with disseminated intravascular coagulation in patients with advanced cancer. J Neurosurg 58:398-401

22. Rhymann FB, Newton WA, Ragab AH (1984) Bone marrow metastases at diagnosis in children and adolescents with rhabdomyosarcoma. Cancer 53:368-373

23. Stéphan JL, Massoubre C, Bertheas MF et al (1996) Fulminant disseminated intravascular coagulation revealing an advanced stage IV poorly differentiated rhabdomyosarcoma. Eur J Pediatr 155:622-623

24. Sakurai JO, Yamada K, Iwasaki M et al (1999) A case of rhabdomyosarcoma of the retroperitoneum in adolescence. Nippon Gekakei Rengo Gakkaishi 24:653-656

25. Yamaguchi K, Koga Y, Suminoe A et al (2007) Alveolar rhabdomyosarcoma of unknown origin mimicking acute leukemia at the initial presentation. Rinsho Ketsueki 48:315-320

26. Scott JP, Morgan E (1983) Coagulopathy of disseminated neuroblastoma. J Pediatr 103:219-222

27. Peterson NE, Galloway B (1982) Wilms tumor with consumption coagulopathy. Urology 19:74-77

28. Muzaffar AR, Friedrich JB, Lu KK et al (2006) Infantile fibrosarcoma of the hand associated with coagulopathy. Plast Reconstr Surg 117:81e-86e

29. Manger DC (1971) Letterer-Siwe's disease: a case complicated by disseminated intravascular coagulation and responding to heparin therapy. Pediatrics 47:435-438

30. Rhymann FB, Newton WA, Ragab AH (1984) Bone marrow metastases at diagnosis in children and adolescents with rhabdomyosarcoma. Cancer 53:368-373

31. Fiegl M, Weltermann A, Stindl R et al (1999) Massive disseminated intravascular coagulation and hyperfibrinolysis in alveolar 
rhabdomyosarcoma: case report and review of the literature. Ann Hematol 78:335-338

32. Stéphan JL, Massoubre C, Bertheas MF et al (1996) Fulminant disseminated intravascular coagulation revealing an advanced stage IV poorly differentiated rhabdomyosarcoma. Eur J Pediatr 155:622-623

33. Tobar A, Avigad S, Zoldan M et al (2000) Clinical relevance of molecular diagnosis in childhood rhabdomyosarcoma. Diagn Mol Pathol 9:9-13

34. Sandberg AA, Stone JF, Czarnecki L et al (2001) Hematologic masquerade of rhabdomyosarcoma. Am J Hematol 68:51-57

35. Reinecke P, Gerharz CD, Thiele KP et al (2000) Temporary remission of an alveolar rhabdomyosarcoma diagnosed and treated as acute leukemia. Leuk Lymphoma 36:405-409

36. Srinivas U, Pillai L, Kar R et al (2007) A case of rhabdomyosarcoma masquerading as acute leukemia at presentation: a case report. Indian J Pathol Microbiol 50:917-919

37. Bozzi F, Collini P, Aiello A et al (2008) Flow cytometric phenotype of rhabdomyosarcoma bone marrow metastatic cells and its implication in differential diagnosis with neuroblastoma. Anticancer Res 28:1565-1569
38. Kussick SJ, Wood BW, Li Y (2001) Using antibodies to CD56 and CD45 to identify nonhematopoietic small blue round cell tumors by flow cytometry [abstract]. Am J Clin Pathol 116:279

39. Bick RL (1992) Coagulation abnormalities in malignancy: a review. Semin Thromb Hemost 18:353-372

40. Weltermann A, Mitterbauer GJ, Mitterbauer M et al (1998) Disseminated intravascular coagulation with excessive fibrinolysis in a patient with metastatic endometrial carcinoma-influence of various treatments on the coagulopathy (a case report). Wien Klin Wochenschr 110:53-57

41. Cerone JR, Kanwar VS, Sills RH (2006) Consumption coagulopathy. Article last updated: 6 Jan 2009

42. Becker JU, Wira CR (2009) Disseminated intravascular coagulation. In: Medscape. http://emedicine.medscape.com/article/ 779097-overview. Accessed 10 Sept 2009

43. Bell WR, Starksen NF, Tong S et al (1985) Trousseau's syndrome. Devastating coagulopathy in the absence of heparin. Am J Med 79:423-430

44. Pernerstorfer T, Hollenstein U, Hansen J et al (1999) Heparin blunts endotoxin-induced coagulation activation. Circulation 100:2485-2490 